\title{
DIGITAL COMPUTER SIMULATION OF HUMAN SYSTEMIC ARTERIAL PULSE WAVE TRANSMISSION: A NONLINEAR MODEL*
}

\author{
BERNARD W. SCHAAF $\dagger$ \\ Bioengineering Program, University of Michigan, Ann Arbor, Michigan 48 104, U.S.A. \\ and \\ PETER H. ABBRECHT \\ Department of Physiology, University of Michigan Medical School, Ann Arbor, \\ Michigan 48104, U.S.A.
}

\begin{abstract}
The transmission of pressure and flow pulse waves in human systemic arteries is modeled using one-dimensional, nonlinear transient analysis on a system of branching, nonuniform tubes. Nonlinearity results from the retention of the vessel cross-sectional area as a dependent variable and from an approximation to the convective acceleration terms.

Coupling the momentum and continuity equations with a linear elastic membrane equation describing the vessel wall yields a system of quasi-linear, hyperbolic partial differential equations, solvable on a digital computer using the method of characteristics and finite difference techniques. Appropriate boundary conditions enabling the application of the model to whole vascular beds are introduced.

Using published data, a reference state for the human arterial system is defined in terms of vessel geometrical and physical parameters. Model behavior in this state is documented at 14 locations corresponding to vascular regions most frequently investigated clinically. Pressure and flow waveforms, and impedances from the model show reasonable agreement with clinical data reported in the literature.

The model is found to reproduce the mechanical behavior of the real system with greater fidelity than previous models. Comparison of the nonlinear model with a linearized, lumped parameter model shows significant differences in performance. These differences are attributed mainly to the nonlinear interaction of vessel transverse and longitudinal impedance. It is concluded that the nonlinear effects of finite vessel wall displacements are of importance in determining arterial pulse propagation behavior, at least in the more distensible central vessels. Fluid friction and convective acceleration effects were found to be of lesser importance in determining overall pulse wave behavior.
\end{abstract}

\section{INTRODUCTION}

THE PURPOSE of this investigation was to develop and verify a rational mathematical model of arterial pulse wave transmission embodying certain nonlinearities of the system. The nonlinear effects of finite vessel wall displacements were of particular interest.

Previous models of arterial systems have been based on linear pulsatile flow theory (Noordergraaf, 1963; de Pater, 1966; Snyder et al., 1968; Westerhof, 1968). Justification for linearization depends partly on whether or not variations of the vessel radius with transmural pressure are 'small' relative to the mean radius. In the stiffer peripheral vessels, this condition is very likely met. However, radial wall displacements on the order of 5-10 per cent of the mean radius can be expected in the human ascending aorta (Patel, 1966), so that the application of linear analysis is questionable.

Two methods have been used in prior efforts to assess the importance of nonlinearities in pulse wave transmission.

*Received 22 June 1971.

†Present address: Department of Engineering Mechanics, Virginia Polytechnic Institute and State University, Blacksburg, Virginia 24061. 
Womersley (1957) investigated convective acceleration and finite wall displacement effects using perturbation analysis on his linear model. Such effects were found to be small. Since the linearized boundary conditions were retained, his results can only give small order corrections to the form of the velocity profile.

One-dimensional fluid mechanical analysis was used by Rockwell et al. (1969) to investigate the formation of shock waves and other nonlinear phenomena in a nonuniform, single tube model of the canine aorta. The nonlinearities were found to have a marked influence on model pressure and flow patterns. Skalak and Stathis (1967) applied linear and one-dimensional nonlinear analysis to a short segment of pulmonary artery. Substantially different results were obtained for the two cases. It was concluded that the differences were primarily due to the finite wall displacements allowed in the nonlinear model.

These studies have served to establish the importance of nonlinear effects in short arterial segments. A goal of the present study was to expand the scope of the system analyzed to assess the overall importance of nonlinearities in an extensive model of the human arterial system.

THE MODEL

\section{One dimensional equations}

The formulation of the model is based on one-dimensional fluid mechanical analysis. The applicability of this approach has been demonstrated in single tube models of arterial flow (Streeter et al., 1963). In using onedimensional analysis, the local detail possible in a linear model (explicit derivation of the fluid velocity profile) is sacrificed in order to obtain a nonlinear description of the gross behavior of pressure and flow. Detailed derivations of the appropriate equations have been given by Streeter et al. (1966), Barnard et al. (1966) and Schaaf (1971) and will not be repeated here.
The derivation is based on the following assumptions (Schaaf, 1971):

(1) An artery can be described as a straight, distensible and slightly tapered tube with a circular cross-section.

(2) Gravity forces can be ignored.

(3) Blood can be considered to be incompressible and to have a linear stress-rate of strain relationship.

(4) The vessel is totally constrained in the longitudinal direction.

(5) There are no secondary flows.

Under these conditions, conservation of fluid mass for the control volume of Fig. 1 is:

$$
\frac{\partial A}{\partial t}+\frac{\partial(A V)}{\partial z}+q=0
$$

where $A$ is the vessel cross-sectional area, $V$ is the mean velocity, $z$ is distance along the axial coordinate, $t$ is time, and $q$ is a term allowing for leakage of fluid through the vessel wall. Such outflow represents the removal of fluid from the primary vessel through small branches. Equation (1) represents the equivalence of the time rate of change of volume

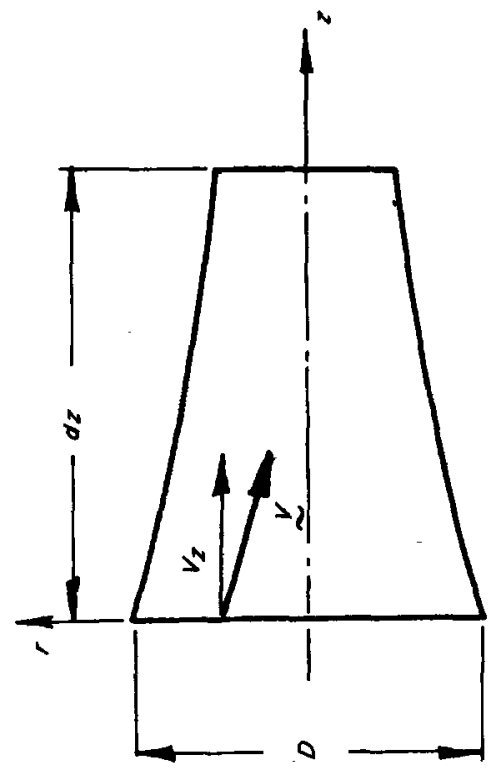

Fig. 1. Sketch of vessel control volume. 
and the net rate of fluid influx per unit length of vessel.

The momentum equation for the same control volume is given by

$$
\begin{array}{r}
\frac{\partial(A V)}{\partial t}+\frac{\partial\left(\beta A V^{2}\right)}{\partial z}+\beta q V+g \gamma A \frac{\partial H}{\partial z}+\frac{1}{\rho} \tau_{0} \pi D \\
=0
\end{array}
$$

where $D$ is the vessel diameter, $H$ is the piezometric head, $g$ is the acceleration due to gravity, $\gamma$ is the ratio of the density of mercury to the density of blood, $\rho$ is the mass density of blood, $\tau_{0}$ is the fluid shear stress evaluated at the vessel wall, and $\beta$ is the one-dimensional momentum flux coefficient accounting for the difference between the square of the mean velocity and the mean value of the local velocity squared, taken over the vessel crosssection. The momentum flux coefficient arises as a consequence of one-dimensionalization. It is defined by

$$
\beta=\frac{1}{A} \int_{A}\left(\frac{v_{z}}{V}\right)^{2} \mathrm{~d} A
$$

where $v_{z}$ is the axial component of $v$.

To obtain (2) it was further assumed that the specific momentum of the leakage outflow, $q$, is the same as that of the primary flow.

Provided the small vessel taper and fluid constitutive assumptions are valid, the wall shear stress can be approximated by

$$
\tau_{0}=-\left.\mu \frac{\partial v_{z}}{\partial r}\right|_{r=D / 2}
$$

where $\mu$ is the dynamic viscosity of blood and $r$ is distance in the radial direction.

In the derivation of (2) and throughout the remainder of this paper, pressures are expressed in units of $\mathrm{mm}$ of mercury rather than in absolute units in order to facilitate comparison of model predictions with data from the literature, most of which are in piezometric units.

In reducing the equations of fluid motion to one-dimensional form, the details concerning the actual shape of the velocity profile are preserved in the parameters $\beta$ and $\tau_{0}$. Since theoretical determination of the parameters would require the solution of the full three-dimensional problem, reasonable values must be approximated. One approach (Barnard, 1966) is to assume a specific formulation for $v_{z}$ and then compute the required parameters using (3) and (4). However, it is worth inquiring under what circumstances this method is likely to improve the model appreciably. We first note that in the limit of very stiff vessel walls, zero lateral outflow and very small taper, the momentum equation reduces to the form valid for a rigid, uniform tube:

$$
\frac{\partial V}{\partial t}+g \gamma \frac{\partial H}{\partial z}+\frac{1}{\rho \bar{A}} \tau_{0} \pi D=0 .
$$

The monentum flux term $\partial\left(\beta A V^{2}\right) / \partial z$ goes to zero while the remaining terms are largely unaffected in approaching this limiting condition. Furthermore, $\beta$ itself cannot reasonably be larger than $1 \cdot 33$, the value for laminar flow. For conditions sufficiently close to the limiting state, it is reasonable to expect the contribution of the momentum flux term to be diminished. On this basis, the momentum flux term is assumed negligible in comparison with the remaining terms. This assumption will be checked later by computing the magnitudes of the individual terms in (2) from the model results.

The contribution of the leakage momentum flux term $\beta q V$ will also be small, since it is used only to represent very small outflows from the primary vessels. The problem is therefore assumed insensitive to the precise value of $\beta$ selected. For mathematical convenience, $\beta$ is assumed to be 1.0 in (2). Multiplying (1) by $V$, subtracting the result from (2) and dividing by $A$ yields the modified momentum equation,

$$
\frac{\partial V}{\partial t}+V \frac{\partial V}{\partial z}+g \gamma \frac{\partial H}{\partial z}+\frac{\pi D}{\rho A} \tau_{0}=0 .
$$

An expression for the wall shear stress is also required. In this case it cannot be argued 
$a$ priori that wall friction is uniformly unimportant. Instead, an approximation derived from the steady-oscillatory flow solution for an infinite, rigid, uniform tube is adopted:

$$
\tau_{0}=\frac{8 \mu}{D} V+\frac{\rho D(\beta-1)}{4} \frac{\partial V}{\partial t} .
$$

Equation (6) relates the wall shear stress to the instantaneous mean flow velocity and to the time rate of change of the velocity. The validity of (6) is restricted to low frequency variations in flow, however the shear stress is overestimated at higher frequencies so that incorporating this approximation will not deemphasize the relative importance of friction effects in the model.

In (6), $\beta$ is assumed equal to $4 / 3$. This is an apparent contradiction to the value used in obtaining equation (5). It should be realized that $\beta$ was selected there as a matter of mathematical convenience to obtain the simpler version of the momentum equation (5). This was possible because of the expected small contribution of the terms in which $\beta$ appeared.

Finally, an equation relating vessel crosssectional area and pressure and an expression for the distributed outflow function must be adopted. Constitutive relations for arterial wall material and the consequent pressure area relationships have been extensively investigated. It has been shown that arterial walls are composed of a material that is to some degree nonlinear viscoelastic and anisotropic (Attinger, 1968). However, it is our opinion that the current state of knowledge of arterial viscoelastic properties is not complete enough to merit their inclusion in an arterial model at the present time. We anticipated that the inclusion of finite radial strains alone would produce significant effects on the wave propagation behavior of the model. The selection of a particular wall model was considered secondary to the admission of finite strains themselves. Under these circumstances, a linear-elastic, in- compressible membrane model of the vessel wall was assumed to be adequate.

For an incompressible elastic wall material (Poisson's ratio $=0.50$ )

$$
D h=D_{0} h_{0}
$$

where $D_{0}$ and $h_{0}$ are the vessel diameter and vessel wall thickness at zero transmural pressure. Using (7) and the method of analysis for stresses within the walls of thinwalled vessels, the following area-pressure relationship is obtained (Streeter et al., 1966):

$$
A=\frac{A_{0}}{1-\frac{g \gamma H \rho D_{0}}{h_{0} E}}=\frac{A_{0}}{1-\frac{g \gamma H}{a_{0}{ }^{2}}}
$$

where $A_{0}$ is the area of the vessel lumen at zero pressure, $E$ is the effective elastic modulus, and $a_{0}=\left(h_{0} E / \rho D_{0}\right)^{0.5}$ is the wave speed. Note that $h_{0}, D_{0}$ and $E$ can vary with location in the system.

A linear dependance on pressure is assumed for the distributed out-flow function,

$$
q=K\left(H-H_{b}\right) .
$$

$K$ is a constant depending only on the location in the system and $H_{b}$ is an assumed constant terminal bed pressure.

Equations (1) and (9) are combined to get

$$
\frac{\partial A}{\partial t}+\frac{\partial(A V)}{\partial z}+K\left(H-H_{b}\right)=0
$$

and (6) is substituted in (5) to get

$$
\beta \frac{\partial V}{\partial t}+V \frac{\partial V}{\partial z}+g \gamma \frac{\partial H}{\partial z}+\frac{8 \pi \nu}{A} V=0
$$

Equations (7-11) then provide the complete one-dimensional system of equations necessary to solve for the dependent variables $A$, $V$, and $H$ in terms of the independent variables $t$ and $z$. The system of equations describes the relationship between pressure, flow and vessel area in a single, nonuniform tube. To obtain the solution for a complex system, appropriate conditions at vessel branches, terminations and the inlet to the system must be introduced. 


\section{BOUNDARY CONDITIONS}

A left ventricular pressure-time function is provided at the inlet to the system. The ventricular pressure is transmitted to the aorta through an idealized aortic valve. The flow through the valve is given by

$$
Q= \pm C \cdot A_{\nu} \sqrt{\Delta H}
$$

where the orifice area, $A_{\nu}$, and the orifice coefficient, $C$, are assumed to be functions of the valve pressure gradient, $\Delta H$. The sign in (12) is selected according to the sign of $\Delta H$. Fig. 2 shows some typical $A_{v}-H$ relations. system, from a simple series continuation of two vessels (i.e. subclavian-axillary) to the branching of a single vessel into three vessels (i.e., celiac-hepatic-gastric-splenic). In the model, these junctions are necessarily represented as idealized structures. Junctions are assumed to have no fluid storage capacity and further, it is assumed that secondary flow effects are negligible. Under these assumptions, the boundary conditions for one vessel branching into $n$ vessels are given by

$$
H_{1}=H_{2}=H_{3}=\cdots=H_{n}
$$

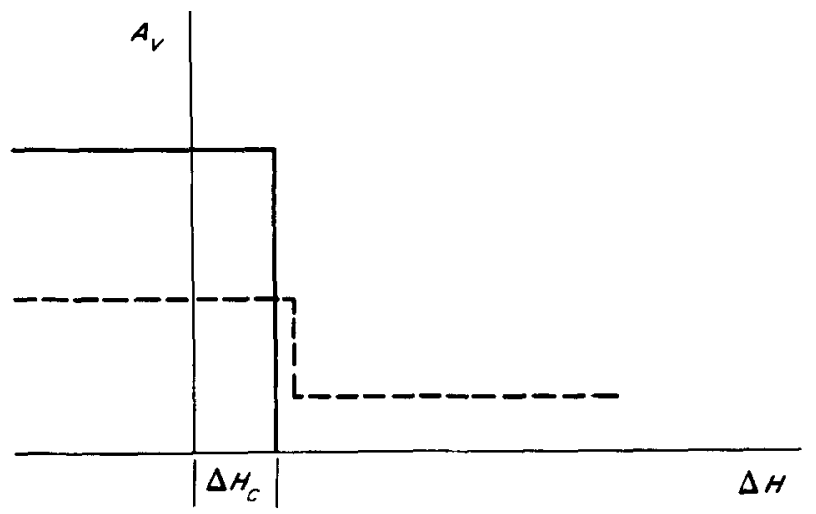

Fig. 2. Aortic valve area functions.

Since the model is an open system describing only the systemic arteries, it is terminated in peripheral lumped parameter loads representing the combined effects of small arteries, arterioles, and capillaries. It is commonly assumed that the terminal loads are purely resistive since the small vessel diameters at this level cause the resistive character of the local impedance to dominate. For a resistive termination, the pressure flow relationship is simply

$$
H-H_{b}=R_{T} Q
$$

where $H_{b}$ is an assumed terminal bed pressure and $R_{T}$ is the lumped resistive load impedance.

Several orders of branching of major arteries can be identified in the arterial expressing the neglect of secondary flows and

$$
\sum_{i=1}^{n+1} A_{i} V_{i}=0
$$

since no fluid is stored in the junction.

The system of equations (7-11) with the appropriate boundary conditions (12-15) is nonlinear and cannot be solved by the usual analytical methods. Instead, it is solved by numerical means, using the method of characteristics and a digital computer (Abbott, 1966; Streeter, 1966; Schaaf, 1971).

\section{MODEL PARAMETERS}

The information fed to the computer consists of geometrical data describing the vessel dimensions and configurations, physical properties of the vessels and the contained 
fluid, initial conditions, driving function data, and program control parameters.

The parameters describing the vessels in the model are the wall thickness, $h_{0}$, diameter, $D_{0}$, the elastic modulus, $E$ and the vessel length, $L$. A fully rational parameter model would use independently measured values for these parameters. In the present study, however, it has been found expedient to present them in the form of the zero-pressure wave speed, $a_{0}$. As sources for these parameters, the authors have drawn on the efforts of previous modelers, notably Noordergraaf (1963) and Westerhof (1968).

Figure 3 is a schematic of the major arteries represented in the model. Although they are not shown in the figure, the arteries of the right arm and leg are also included. Symmetry is assumed for certain portions of the system.

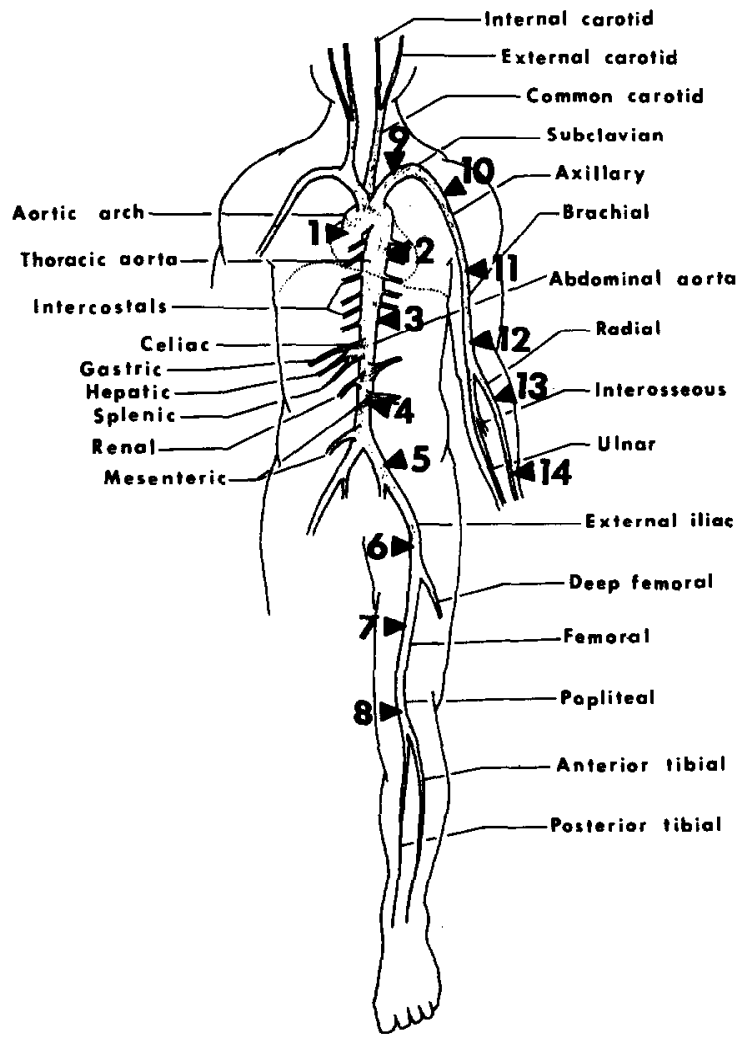

Fig. 3. Schematic of model arterial system showing major arteries included and reference output locations.
Examples are the renal arteries and the arteries of the legs. The intercostals, shown as discrete vessels, are actually modeled as a distributed outflow from the thoracic aorta. Values of $L, D_{0}$, and $a_{0}$ for the proximal and distal ends of each of the arteries are given in Table 1. These quantities are assumed to vary linearly over the specified vessel segment.

Initial conditions on pressure and velocity are supplied as input data for all locations in the modeled system. The initial conditions are those which would exist for a non-oscillatory flow equivalent to the expected cardiac output. The conditions in individual vessels are apportioned according to the individual terminal resistance parameters. Terminal resistances are calculated from the specified total peripheral resistance and the distribution of flow estimated from physiological data. Starting the computations from this steady flow condition, the solution converges to a steady-oscillatory state within three pulse cycles.

Values of the terminal resistances were estimated on the basis of a total parallel combination of $1.04 \mathrm{~mm} \mathrm{Hg} / \mathrm{ml} / \mathrm{sec}$. Notice that this is not quite the normal total peripheral resistance $\quad(T P R=100 \mathrm{~mm} \mathrm{Hg} / 5000 \mathrm{ml} / 60$ $\mathrm{sec}=1.2 \mathrm{~mm} \mathrm{Hg} / \mathrm{ml} / \mathrm{sec}$ ) since it does not include the series frictional resistance in the vessels, which will vary with the flow conditions. The model terminal resistances used are given in Table 2.

Additional specified parameters are: a heart rate of 75 beats $/ \mathrm{min}\left(f_{1}=1.25 \mathrm{c} / \mathrm{s}\right.$, $T=0.8 \mathrm{sec}$.), terminal and distributed outflow bed pressures of $0.0 \mathrm{~mm} \mathrm{Hg}$ and an aortic valve area of $7 \cdot 6 \mathrm{~cm}^{2}$.

\section{RESULTS}

The primary output from the simulation program consists of pressure and flow rate data at up to 14 locations in the model. The output locations are specified by an identifying number and distance from the aortic valve in Table 3 and are shown schematically in Fig. 3. 
Table 1. Model parameters

\begin{tabular}{|c|c|c|c|c|c|c|}
\hline \multirow{2}{*}{$\begin{array}{c}\text { Vessel } \\
\text { No. }\end{array}$} & \multirow[b]{2}{*}{ Description } & \multirow{2}{*}{$\begin{array}{l}\text { Length, } L \\
\text { (cm) }\end{array}$} & \multicolumn{2}{|c|}{$\begin{array}{c}\text { Diameter, } D_{0} \\
(\mathrm{~cm})\end{array}$} & \multicolumn{2}{|c|}{$\begin{array}{c}\text { wave speed, } a_{0} \\
(\mathrm{~cm} / \mathrm{sec})\end{array}$} \\
\hline & & & Proximal & Distal & Proximal & Distal \\
\hline 1 & Asc.-des. aorta & $6 \cdot 3$ & $2 \cdot 340$ & $1 \cdot 810$ & 550 & 550 \\
\hline 2 & Thoracic aorta & $20 \cdot 1$ & $1 \cdot 810$ & 0.938 & 550 & 575 \\
\hline 3 & Abdominal aorta & $5 \cdot 2$ & 0.938 & 0.938 & 575 & 575 \\
\hline 4 & Abdominal aorta & $2 \cdot 5$ & 0.938 & 0.923 & 575 & 577 \\
\hline 5 & Abdominal aorta & $9 \cdot 2$ & 0.923 & 0.856 & 577 & 585 \\
\hline 6,36 & Illiac-prox. femoral & $21 \cdot 0$ & 0.604 & 0.424 & 678 & 717 \\
\hline 7,38 & Femoral & $25 \cdot 7$ & 0.432 & 0.405 & 717 & 734 \\
\hline 8,39 & Popliteal & 17.9 & 0.416 & $0 \cdot 321$ & 977 & 1000 \\
\hline 9,41 & Post. tibial & 29.8 & 0.443 & 0.246 & 1275 & 1470 \\
\hline 10,40 & Ant. tibial & $32 \cdot 6$ & 0.239 & $0 \cdot 246$ & 1480 & 1480 \\
\hline 11,37 & Deep femoral & $15 \cdot 4$ & 0.497 & 0.359 & 1200 & 1200 \\
\hline 12 & Inferior mesenteric & $5 \cdot 9$ & 0.284 & 0.284 & 700 & 700 \\
\hline 13 & Superior mesenteric & $7 \cdot 4$ & 0.700 & 0.700 & 600 & 600 \\
\hline 14,35 & Renal & $3 \cdot 0$ & 0.448 & 0.448 & 700 & 700 \\
\hline 15 & Celiac & $2 \cdot 5$ & 0.625 & 0.625 & 600 & 600 \\
\hline 16 & Splenic. & $7 \cdot 4$ & 0.440 & 0.440 & 600 & 600 \\
\hline 17 & Hépatite. & $7 \cdot 4$ & $0 \cdot 353$ & 0.353 & 600 & 600 \\
\hline 18 & Gastric & $7 \cdot 4$ & $0 \cdot 289$ & 0.289 & 600 & 600 \\
\hline 19,34 & Anonymal & $6 \cdot 4$ & $1 \cdot 100$ & 0.750 & 800 & 800 \\
\hline 20,42 & Subclavian-axillary & $19 \cdot 9$ & 0.750 & 0.541 & 800 & 800 \\
\hline 21,43 & Brachial & $25 \cdot 5$ & 0.552 & 0.432 & 925 & 1065 \\
\hline 22,45 & Radial & $24 \cdot 5$ & $0 \cdot 340$ & $0 \cdot 264$ & 1065 & 1200 \\
\hline 23,44 & Prox. ulnar & $4 \cdot 9$ & 0.405 & 0.394 & 1065 & 1174 \\
\hline 24,47 & Dist. ulnar & $20 \cdot 4$ & $0 \cdot 394$ & $0 \cdot 350$ & 1174 & 1200 \\
\hline 25,46 & Interosseous & 8.6 & $0 \cdot 171$ & $0 \cdot 171$ & 1000 & 1000 \\
\hline 26,33 & Vertebral - & $17 \cdot 8$ & $0 \cdot 350$ & 0.350 & 1000 & 1000 \\
\hline 27,32 & Com. carotid & $3 \cdot 7$ & 0.581 & 0.620 & 575 & 600 \\
\hline $28,29,30,31$ & Ext.int carotid & $5 \cdot 4$ & $0 \cdot 318$ & $0 \cdot 160$ & 800 & 1050 \\
\hline
\end{tabular}

Table 2. Terminal resistances

\begin{tabular}{clc}
\hline $\begin{array}{c}\text { Vessel } \\
\text { No. }\end{array}$ & Description & $\begin{array}{c}\text { Terminal } \\
\text { resistance } \\
\text { (mm Hg/ml/sec) }\end{array}$ \\
\hline 9,41 & Post. tibial & $35 \cdot 85$ \\
10,41 & Ant. tibial & $42 \cdot 00$ \\
11,37 & Deep femoral & $35 \cdot 85$ \\
12 & Inf. mesenteric & $51 \cdot 74$ \\
13 & Sup. mesenteric & $7 \cdot 00$ \\
14,35 & Renal & $8 \cdot 55$ \\
16 & Splenic & $17 \cdot 47$ \\
17 & Hepatic & $27 \cdot 29$ \\
18 & Gastric & $40 \cdot 64$ \\
22,45 & Radial & $39 \cdot 70$ \\
24,47 & Ulnar & $39 \cdot 70$ \\
25,46 & Interosseous & $633 \cdot 6$ \\
26,33 & Vertebral & $45 \cdot 22$ \\
28,29 & Ext. carotid & $104 \cdot 3$ \\
30,31 & Int. carotid & $104 \cdot 3$ \\
$2 *$ & Intercostals & $10 \cdot 46$ \\
\hline
\end{tabular}

*As distributed outflow from thoracic aorta.
Table 3. Model output locations

\begin{tabular}{ccl}
$\begin{array}{c}\text { Location* } \\
\text { No. }\end{array}$ & $\begin{array}{c}\text { Distance } \\
\text { from aortic } \\
\text { valve (cm) }\end{array}$ & \multicolumn{1}{c}{ Vessel } \\
\hline 1 & 0 & Origin of asc. aorta \\
2 & $8 \cdot 5$ & Prox. thoracic aorta \\
3 & $19 \cdot 6$ & Dist. thoracic aorta \\
4 & $32 \cdot 1$ & Dist. abdominal aorta \\
5 & $44 \cdot 2$ & Illiac \\
6 & $56 \cdot 1$ & Prox. femoral \\
7 & $72 \cdot 1$ & Dist. femoral \\
8 & $89 \cdot 4$ & Prox. popliteal \\
9 & $16 \cdot 3$ & Subclavian \\
10 & $26 \cdot 2$ & Axillary \\
11 & $36 \cdot 9$ & Prox. brachial \\
12 & $49 \cdot 5$ & Dist. brachial \\
13 & $63 \cdot 1$ & Prox. radial \\
14 & $77 \cdot 8$ & Dist. radial \\
\hline
\end{tabular}

*See Fig. 3 for schematic of these output sites. 
These particular output sites were selected to correspond to the segments of the vasculature most frequently investigated in clinical situations.

Predicted aortic root flow and pressure for a ventricular pressure forcing function are shown in Fig. 4. The ventricular pulse was obtained from a clinical tracing (Wiggers, 1952). The time axis in Fig. 4 has been normalized with respect to the period of the heart beat, $T$. Pressure and flow waveforms for all output locations are displayed in Figs. 5 and 6.

The model ventricular flow ejection pulse in Fig. 4 conforms well with the pulse wave form measured by Spencer and Denison (1959) shown as the dashed line in the same
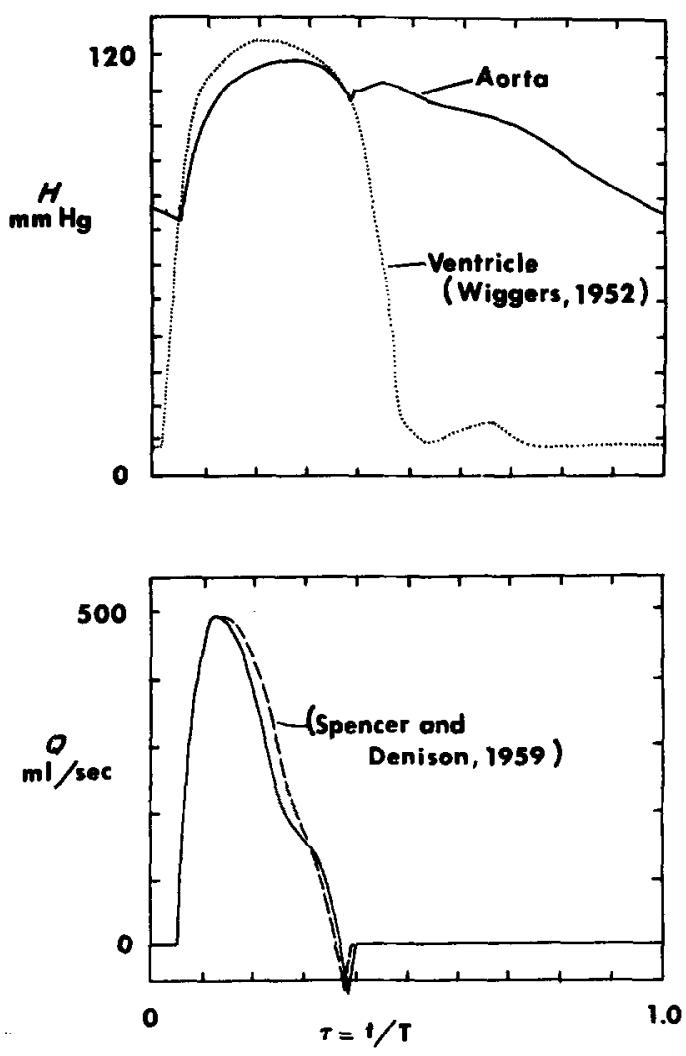

Fig. 4. Model response at root of aorta (Site 1) to ventricular pressure forcing function. Ventricular pressure pulse from Wiggers (1952). Clinical flow pulse from Spencer and Denison (1959).
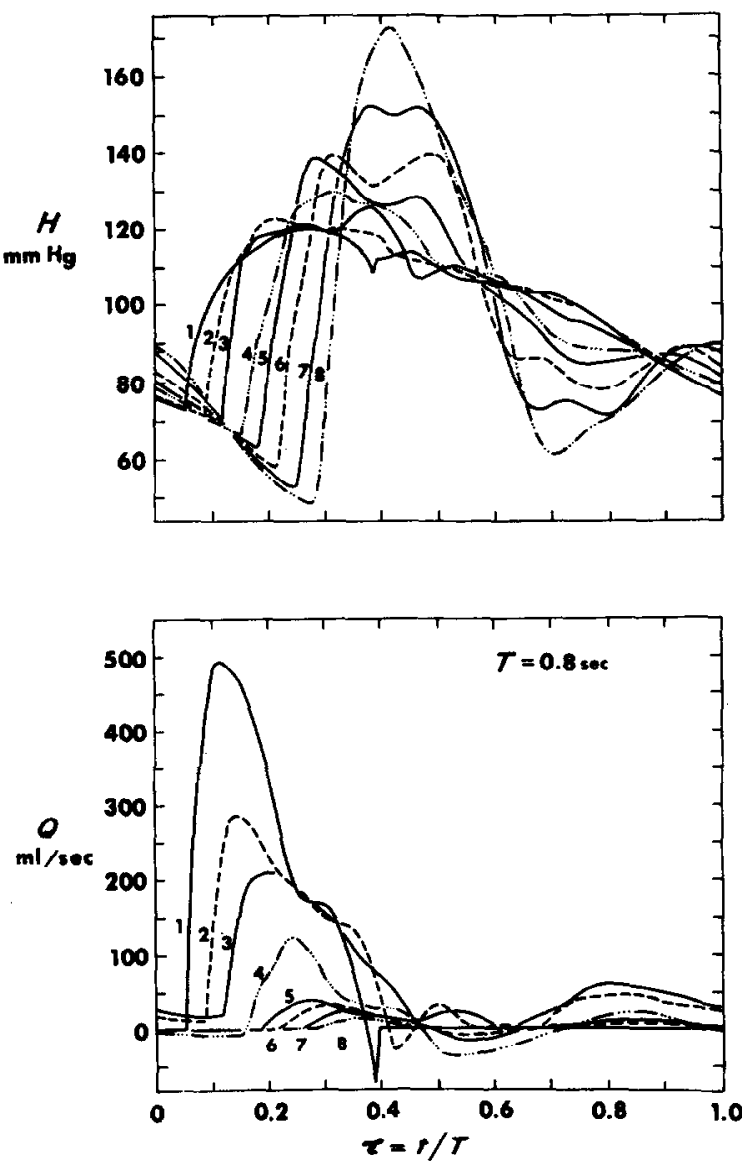

Fig. 5. Pressure and flow pulse waveforms in the model aorta-popliteal channel. Numbers refer to output locations. (See Table 3).

figure. Peak systolic flows are the same (about $500 \mathrm{ml} / \mathrm{sec}$ ) and the retrograde flow maximums coincide. The inflection in the descending limb of the measured pulse is less pronounced than that seen in the model pulse. Cardiac output in the model is $5.11 . / \mathrm{min}$ compared to $5 \cdot 3 \mathrm{l} . / \mathrm{min}$ for the clinical pulse.

Pressure pulse mappings from the model are replotted in Fig. 7 for the aorta-popliteal channel and Fig. 8 for the subclavian-radial channel along with clinically obtained measurements (Kroeker and Wood, 1955; O'Rourke et al., 1968). Behavior of the model pulses is similar to the clinical results. Those features and transformations of the pulse 

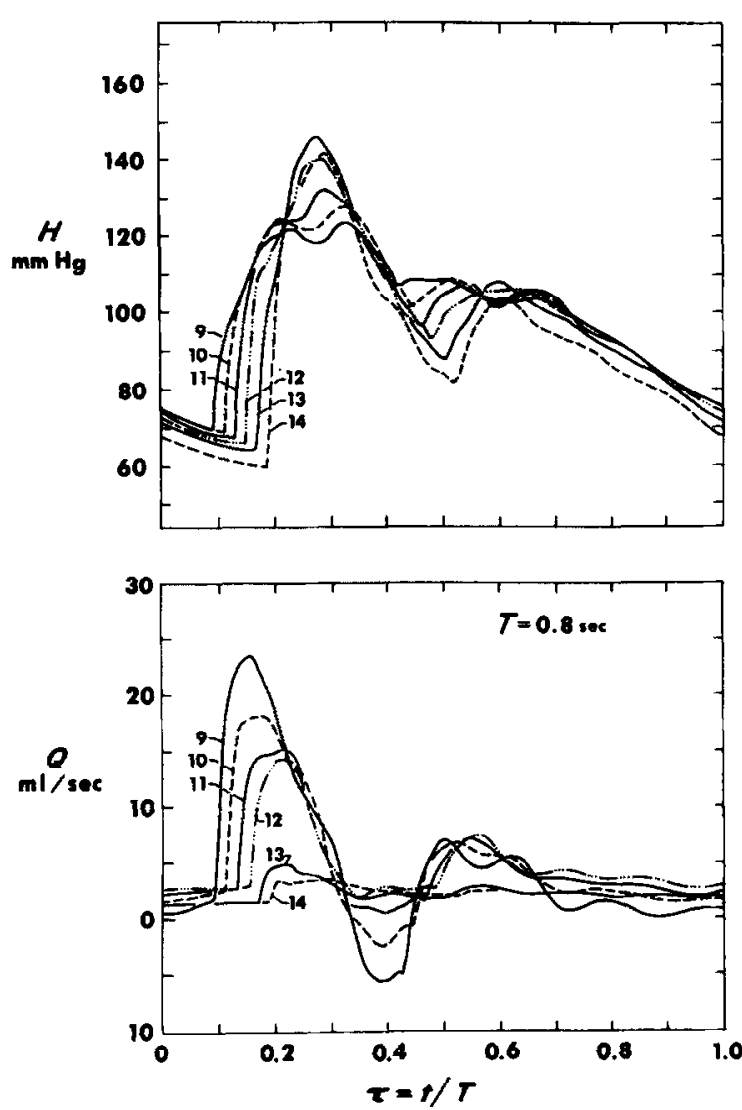

Fig. 6. Pressure and flow pulse waveforms in the model subclavian-radial channel. Numbers refer to output locations. (See Table 3). usually observed are clearly evident in both sets of curves.

Concise presentation of pressure-flowfrequency relationships in arterial flow can be made using the concept of impedance. Given the complex Fourier series for the pressure and flow pulses,

$$
Q(t)=Q_{0}+\sum_{n=1}^{\infty} Q_{n} e^{i\left(n \omega t-\alpha_{n}\right)}
$$

and

$$
H(t)=H_{0}+\sum_{n=1}^{\infty} H_{n} e^{i\left(n \omega t-\beta_{n}\right)}
$$

where $\omega$ is the fundamental angular frequency, $n$ is the harmonic number, and $\alpha_{n}$ and $\beta_{n}$ are the relative phase angles of the individual components, the complex local impedance is defined by

$$
Z_{n}=\left|H_{n} / Q_{n}\right| e^{i \phi_{n}}
$$

where $\phi_{n}=\beta_{n}-\alpha_{n}$. It is customary to present plots of the impedance magnitude, $H_{n} / Q_{n}$ and the phase angles, $\phi_{n}$, as functions of frequency. The dominant nature of complicated systems can be inferred from such plots by comparison with the known form of impedance plots for simpler systems.

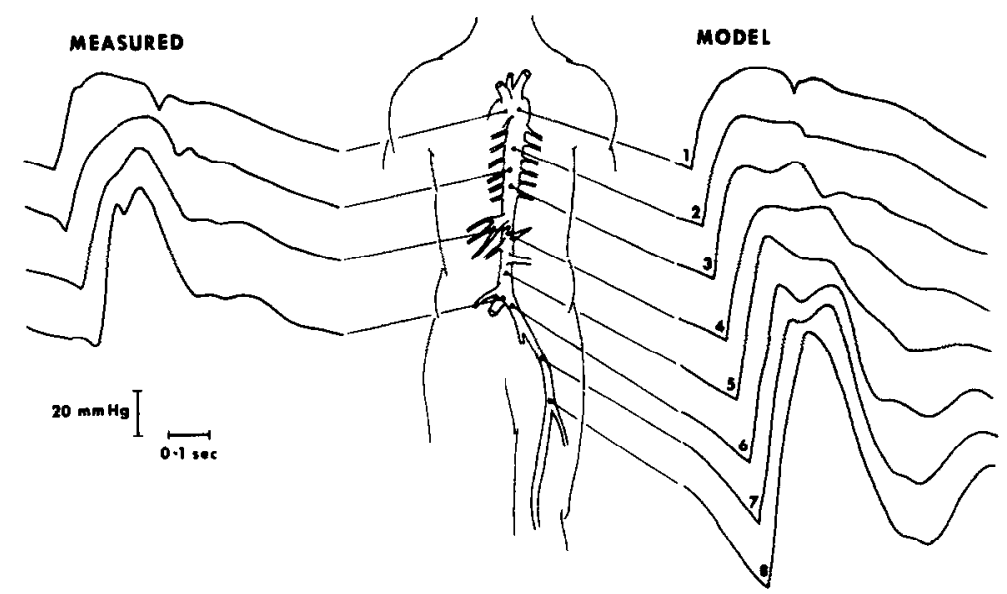

Fig. 7. Comparison of model and clinically measured (O'Rouke et al., 1968) pressure pulse waveforms in aorta. Schematic locations are approximate, exact locations for model waveforms are given in Table 3 . 


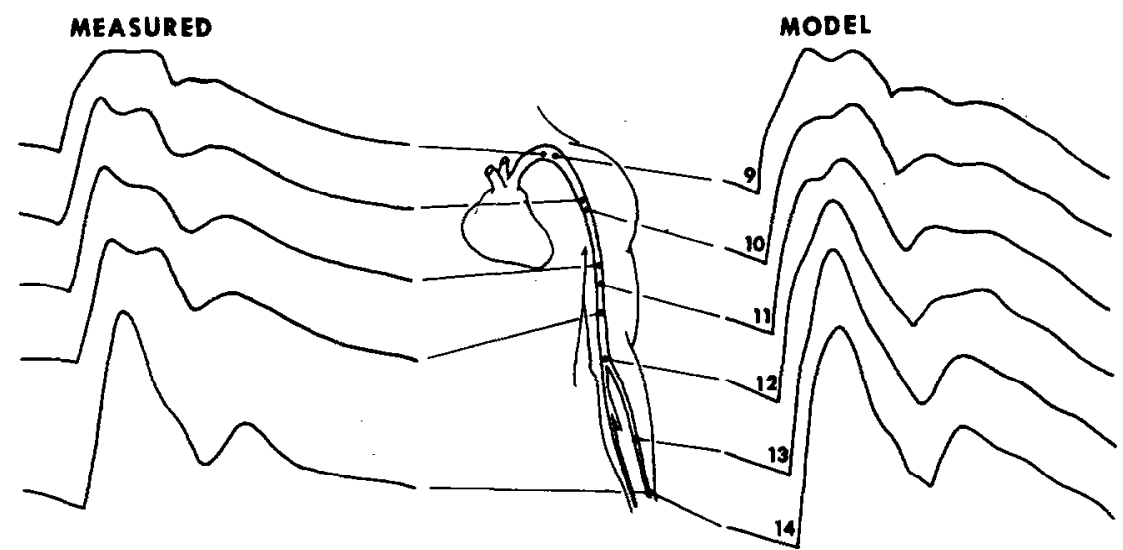

$20 \mathrm{~mm} \mathrm{Hg}] \underset{0.1 \mathrm{sec}}{\longmapsto}$

Fig. 8. Comparison of model and clinically measured (Kroeker et al., 1955) pressure pulse waveforms in the subclavian-radial channel. Schematic locations are approximate, exact locations for model waveforms are given in Table 3.
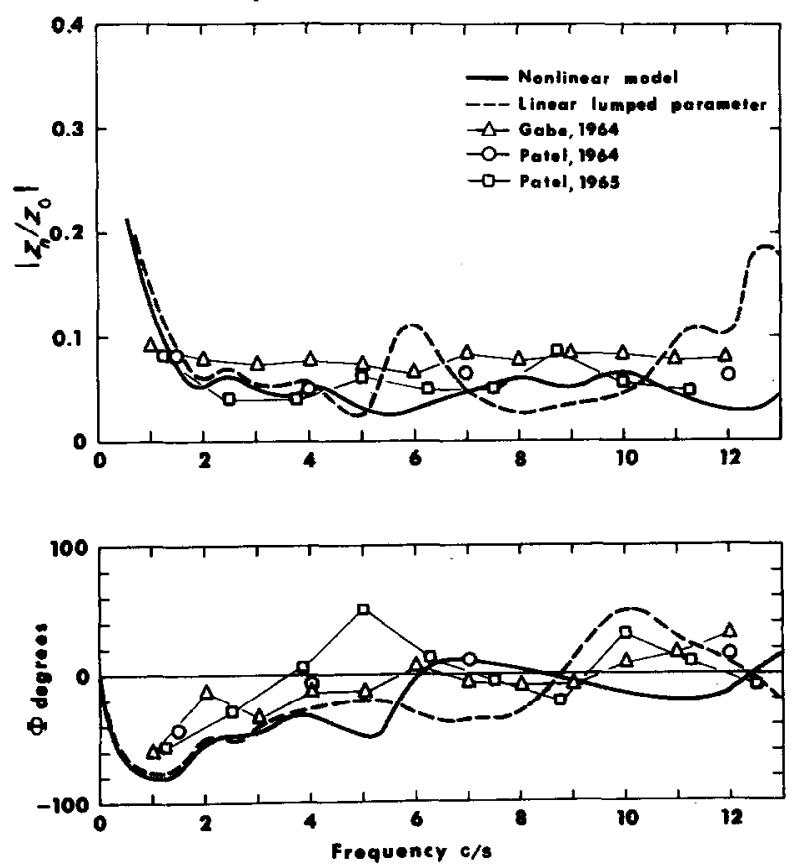

Fig. 9. Normalized input impedance at root of aorta (Site 1) for nonlinear model, a lumped parameter linear model and clinically obtained values in man. Total peripheral resistance for the models is $1.15 \mathrm{~mm} \mathrm{Hg} / \mathrm{ml} / \mathrm{sec}$. 
Impedance measurements in man are available only for the ascending aorta and femoral arteries, sites commonly accessible during cardiovascular diagnostic and surgical procedures. Model and clinically obtained impedances (Gabe et al., 1964; Patel et al., 1964; Patel et al., 1965) for these two locations are shown in Figs. 9 and 10. The model impedance plots are drawn as smooth functions of frequency; while Fourier analysis can actually only yield values of the impedance at discrete multiples of the fundamental frequency. Continuous curves were obtained by operating the model at many different fundamental frequencies and joining the closely spaced results with a smooth line. The impedance magnitudes were normalized by dividing by the local vascular resistance, $Z_{0}=H_{0} / Q_{0}$.

Each symbol in Figs. 9 and 10 represents measurements from a single individual, except the circles in Fig. 9 which are average values from three subjects (Patel et al., 1964). Both model and measured aortic impedance magnitudes are relatively independent of frequency. The magnitudes are in reasonable agreement. At low frequencies, the model aortic impedance phase angles are somewhat more negative than the clinical values. A general positive trend for the phase is seen in both the model and measured results.

In the femoral artery, agreement of model and measurements is not so obvious at first glance because of the wide variations between the measurements on individuals. However, there are qualitative similarities between the model predictions and the experimental data. Each of the individual clinical curves shows a relative minimum between 2 and $4 \mathrm{c} / \mathrm{s}$ and a relative maximum between 4.5 and $7 \mathrm{c} / \mathrm{s}$. A minimum at about $3.3 \mathrm{c} / \mathrm{s}$ and a relative maximum at $5.75 \mathrm{c} / \mathrm{s}$ for the model can be accepted as reasonable agreement in this respect, particularly in view of the wide differences
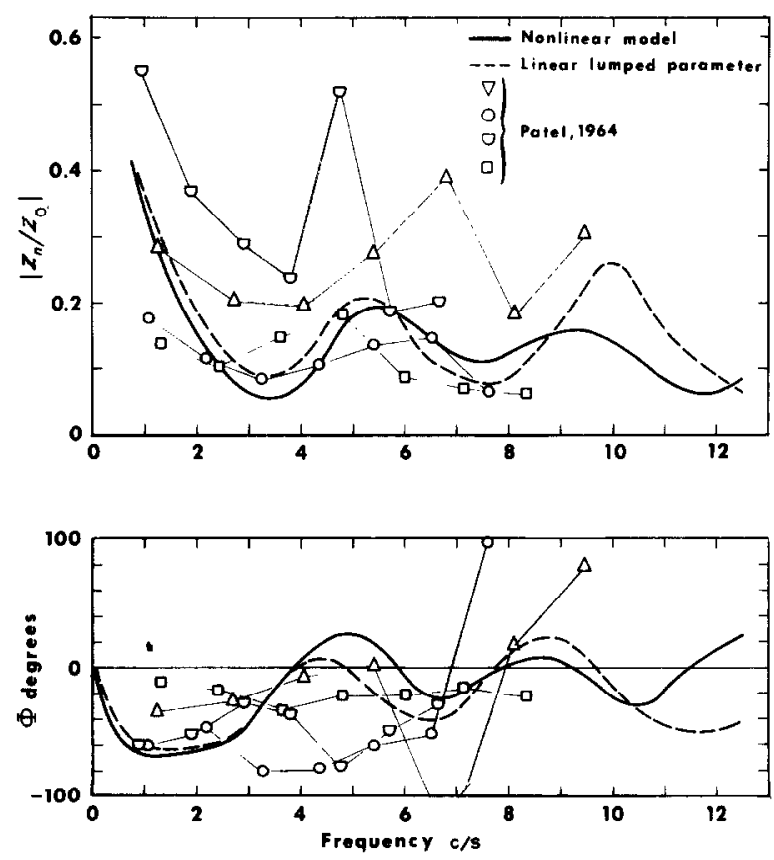

Fig. 10. Normalized input impedance of femoral artery (Site 6) for nonlinear model, a linear, lumped parameter model and clinically obtained values in man. Vascular resistance for the models is $15.3 \mathrm{~mm} \mathrm{Hg} / \mathrm{ml} / \mathrm{sec}$. 
displayed for the measured values. Variations in relative magnitudes with frequency for the model fall within the extremes exhibited by the measured curves. Phase angles measured clinically at this location do not behave in a discernibly regular way except for being uniformly negative below about $6 \mathrm{c} / \mathrm{s}$, and thus are not adequate for testing the model.

\section{DISCUSSION}

In general, the nonlinear model predicts the pulse wave behavior of the real arterial system with reasonable fidelity. In particular, the dominant aspects of pulse wave distortion, such as pulse pressure amplification and the formation of a secondary wave as the pulse is transmitted distally, are well reproduced.

In the model, the pulse pressure widens by about 70 per cent from the aortic arch to the illiac artery. The clinical results also show an amplification of around 70 per cent (Fig. 7). In the same clinical study (O'Rourke et al., 1968), an average amplification of 55 per cent, with a range of about 30-90 per cent, was found for eleven subjects under age 15 . With increasing age, a marked reduction of amplification was indicated. Kroeker and Wood
(1955) reported a smaller average aorta-illiac amplification of 39 per cent for twelve healthy subjects with a range of 18-70 per cent. Amplification in the subclavian-radial channel is approximately 80 per cent for the clinical results shown in Fig. 8 and 50 per cent for the model. Measurements over the same region have been reported to range from 1992 with an average of 46 per cent (Kroeker and Wood, 1955).

An interesting similarity in the primary wave of the model and clinical pulses is shown both in the aorta and in the upper extremity vessels. A prominent biphasic peak develops in the distal thoracic aorta and proximally in the subclavian artery. Although this is seen in almost all extensive pulse mappings, it has not been remarked upon in discussions of the formation of the arterial pulse waveform.

While the nonlinear model is successful in simulating human arterial pulse wave transmission, it is relevant to ask if this effort represents in some measure an improvement over already existing models. This question can be investigated through a direct comparison of the nonlinear model results with the behavior of a linearized, lumped parameter model.

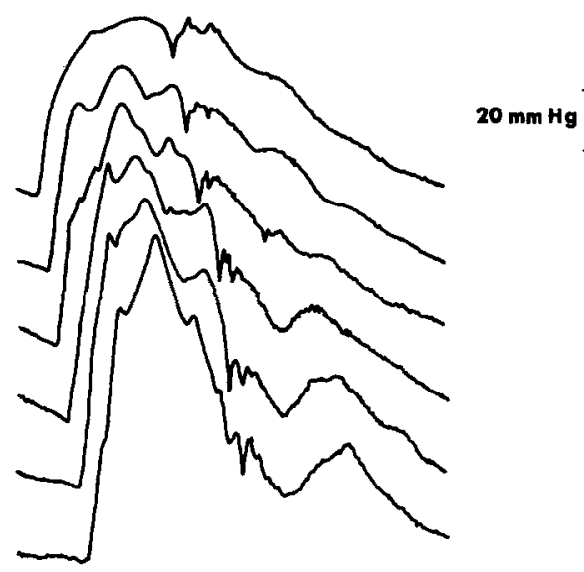

AORTA

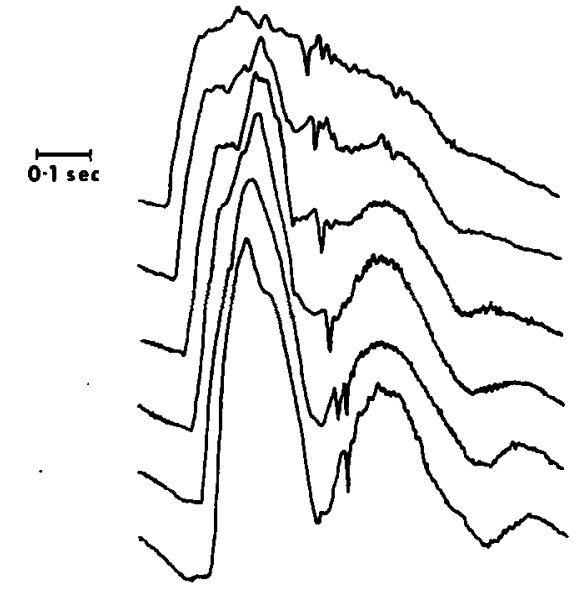

ARM

Fig. 11. Pressure pulse mappings in the aorta and the subclavian-radial arteries for the linear, lumped parameter model. 
Equations (10) and (11) can be linearized to obtain (Taylor, 1965)

$$
\mathscr{L} \frac{\partial Q}{\partial t}+g \gamma \frac{\partial H}{\partial z}+\mathscr{W} Q=0
$$

and

$$
\mathscr{C} \frac{\partial H}{\partial t}+\frac{\partial Q}{\partial z}+q=0
$$

where $\mathscr{L}$ is the inertiance per unit length of vessel, $\mathscr{C}$ is the compliance per unit length, $\mathscr{W}$ is the fluid resistance per unit length and $q$ is the leakage per unit length. Values for these linear parameters are derived from the information in Table 1 , assuming a mean arterial pressure of $100 \mathrm{~mm} \mathrm{Hg}$ and using the definitions

$$
\mathscr{C}=\left.\frac{\partial A}{\partial H}\right|_{H=H_{0}}
$$

and

$$
\mathscr{L}=\beta \frac{\rho}{\bar{A}}
$$

where $\bar{A}$ is the area at the assumed mean pressure, $H_{0}$.

Equations (6) for the wall shear stress and (9) for the distributed outflow are retained.

The linear system thus defined can be solved using electric or hydraulic transmission line techniques in the frequency domain with a digital computer, but to provide a more direct comparison, the method of characteristics was used to obtain the solution. Vessel properties were lumped (held constant) over $\Delta z$ reaches determined by the finite difference characteristic line equation for the linear system given by (Schaaf, 1971).

$$
\frac{\Delta z}{\Delta t}=\frac{1}{T \beta} \sqrt{\frac{\beta}{\mathscr{L} \mathscr{C}}} .
$$

Segment lengths for a $\Delta t$ of 0.005 then range from about $2 \mathrm{~cm}-4.5 \mathrm{~cm}$ in the linear model.

Pulse wave mappings from the linear model, driven by the ventricular pulse of Fig. 4, are shown in Fig. 11 for the aorta-femoral arteries and the arteries of the arm. The gross behavior of the pulse as it propagates in this model conforms to that observed in the real system. Yet, comparison with pulse mappings from the nonlinear model displayed in Figs. 7 and 8 shows that the two dynamic representations of the same physical parameters yield quite different results. An obvious difference is in the response to higher frequency disturbances. The incisura in the nonlinear model, once formed, is quickly damped in transmission through the aorta. In the linear model, the events causing the incisura give rise to high frequency local vibrations which are then propagated throughout the model, apparently without attenuation. Lower frequency oscillations also occur in the linear model which are absent from the nonlinear model. In short, the linear representation, under the same conditions and solution method, is a good deal noisier. Careful examination of the analytical results reported by other investigators using linear models (Westerhof, 1968) also reveals the presence of more noise than in the nonlinear model.

Linear model input impedances for the aorta and the femoral artery are shown in Figs. 9 and 10. Below about $4 \mathrm{c} / \mathrm{s}$ there is little difference between the two models. In the aorta, considerably more oscillation of the input impedance with frequency is seen with the linear model for the midrange frequencies. It can be said that, at this location, the impedance load given by the nonlinear model more nearly resembles the physiological measurements than does that given by the linear model. Results in the femoral artery do not allow this claim. Again, impedance values for both models are very nearly the same for frequencies below $4 \mathrm{c} / \mathrm{s}$. The difference between the models at higher frequencies is not as severe as in the aorta. Furthermore, there is little reason to favor either model on the basis of femoral artery impedance comparisons with physiological measurements. 
The unique features in the nonlinear model are the retention of vessel cross-sectional area as a dependent variable, the inclusion of an approximation to the convective accleration effects (momentum flux terms) and the higher order of approximation to a continuous system.

In deriving the one-dimension model equations, the momentum flux term in the momentum equation was assumed to be small. To test the validity of this assumption, the model pressure and flow results were used to compute the magnitudes of the individual terms of the momentum equation over one pulse cycle. Inertia, momentum flux, and pressure gradient terms from those computations are shown in Figs. 12 and 13 for the proximal thoracic aorta (site 2) and for the proximal radial artery (site 13 ) respectively. At both locations the momentum flux term is indeed small compared to the others.

Figure 14, a pulse mapping for the model with the momentum flux terms ignored, provides further confirmation of the unimportance of convective acceleration effects. There are only small differences in the pulse waveforms with or without the convective acceleration terms. In Figs. 15 and 16, the input impedances for the complete model and the model without the convective acceleration terms are compared for the aorta and the femoral artery. Again, the differences seen are small and it can be concluded that convective acceleration effects are relatively unimportant in determining the gross pulse propagation behavior of the model and therefore are unlikely to be of importance in the real system. This fundamental assumption, based on order of magnitude arguments, is common to all linear analyses of the arterial system. (Womersley, 1955; Noordergraaf, 1968). It is clear that the assumption is valid and furthermore, that the convective acceleration terms cannot account for the differences between the nonlinear model and the linear, lumped parameter model.

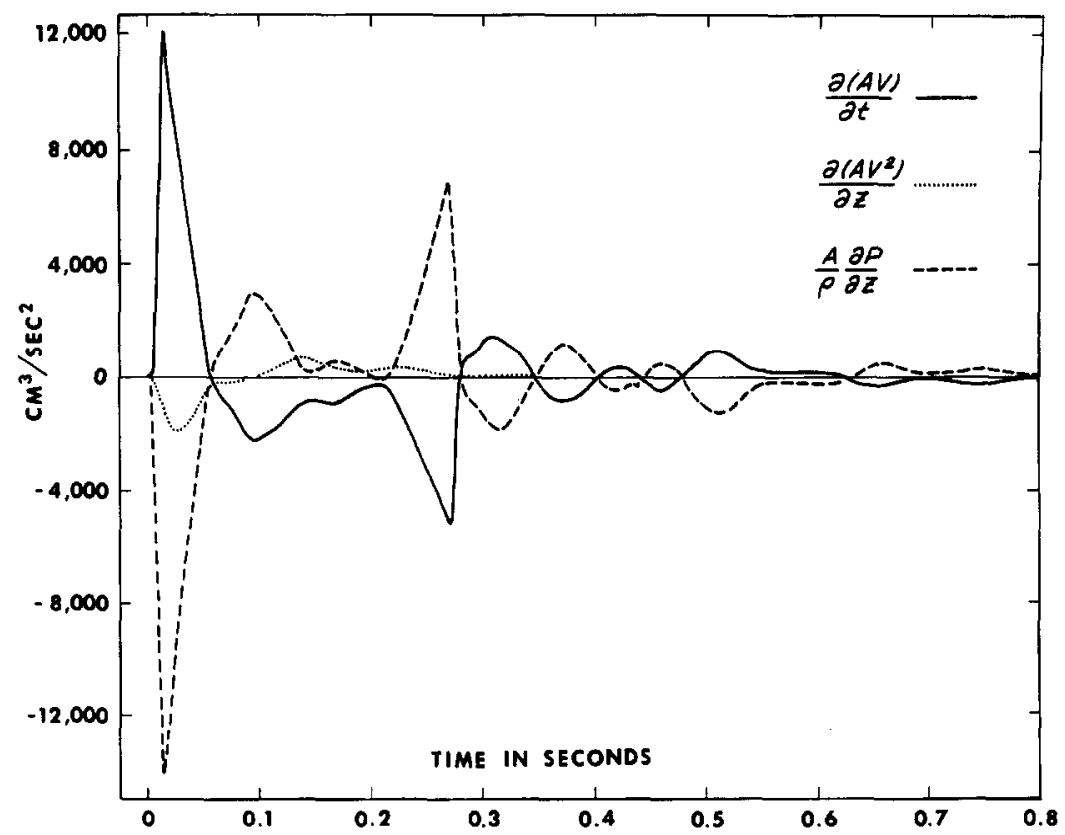

Fig. 12. Magnitudes of the inertia, momentum flux, and pressure gradient terms in the momentum equation (2) over one pulse cycle for the proximal thoracic aorta (Site 2). 


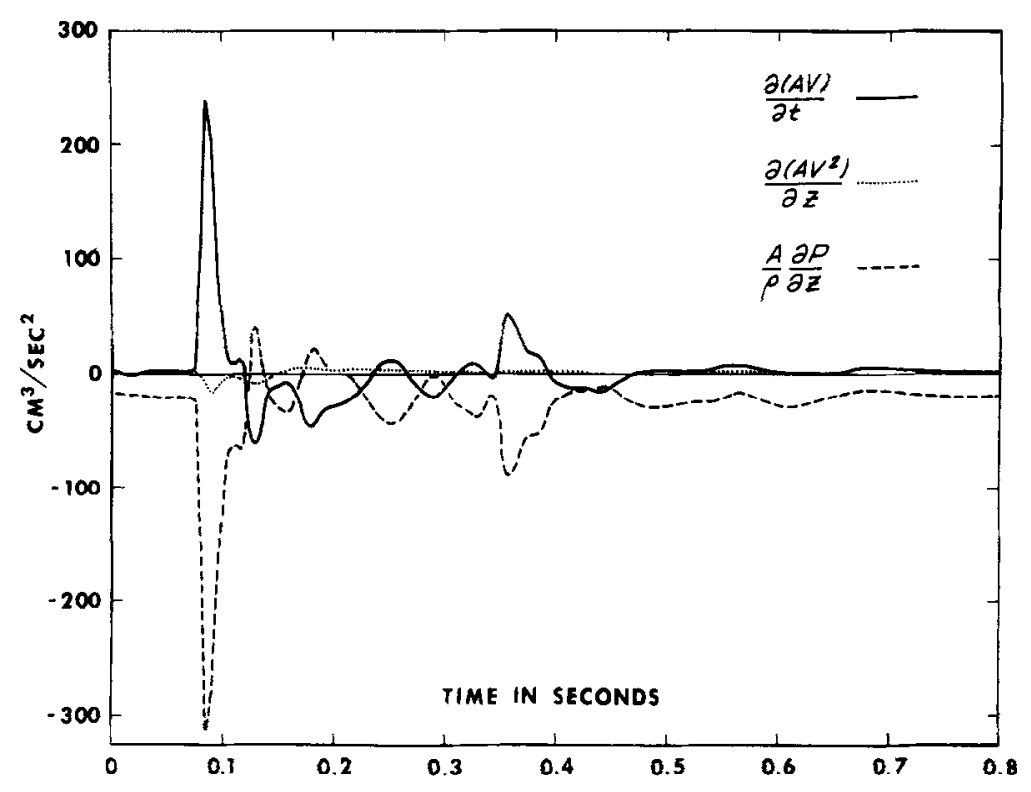

Fig. 13. Magnitudes of the inertia, momentum flux, and pressure gradient terms in the momentum equation (2) over one pulse cycle for the proximal radial artery (Site 13).
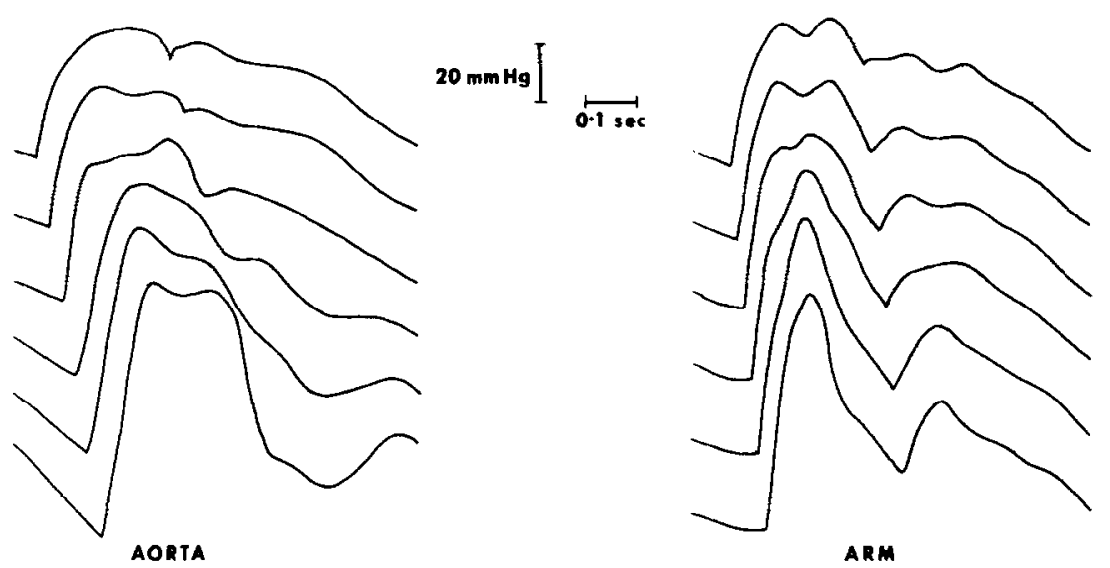

Fig. 14. Pressure pulse mappings in the aorta and subclavian-radial arteries for the nonlinear model, ignoring the convective acceleration terms.

Much attention has been given in the linear modeling of arterial beds to the interaction between fluid inertiance and the wall frictional resistance through the so-called 'sleeve effect'. Recognizing that the assumption of a quasisteady, laminar (parabolic) velocity profile is not realistic, a more accurate representation of the velocity profile, and therefore, the wall shear stress, is sought using oscillatory flow theory (Jager et al., 1966). In passive analogs, the introduction of oscillatory flow theory gives rise to 'corrective' networks for the longitudinal impedance elements, which are claimed to improve the performance of the passive analog model (Westerhof, 1968). In view of these efforts, it is of interest to 

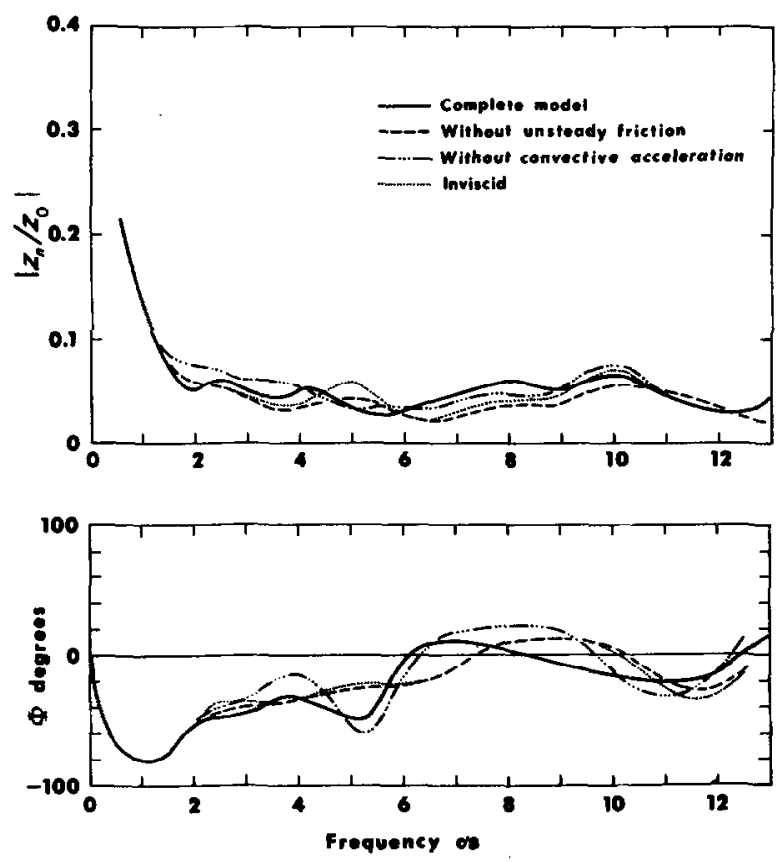

Fig. 15. Normalized input impedance of root of aorta (Site 1), comparing the effects of ignoring unsteady fluid friction, all fluid friction effects (inviscid case) and convective acceleration.
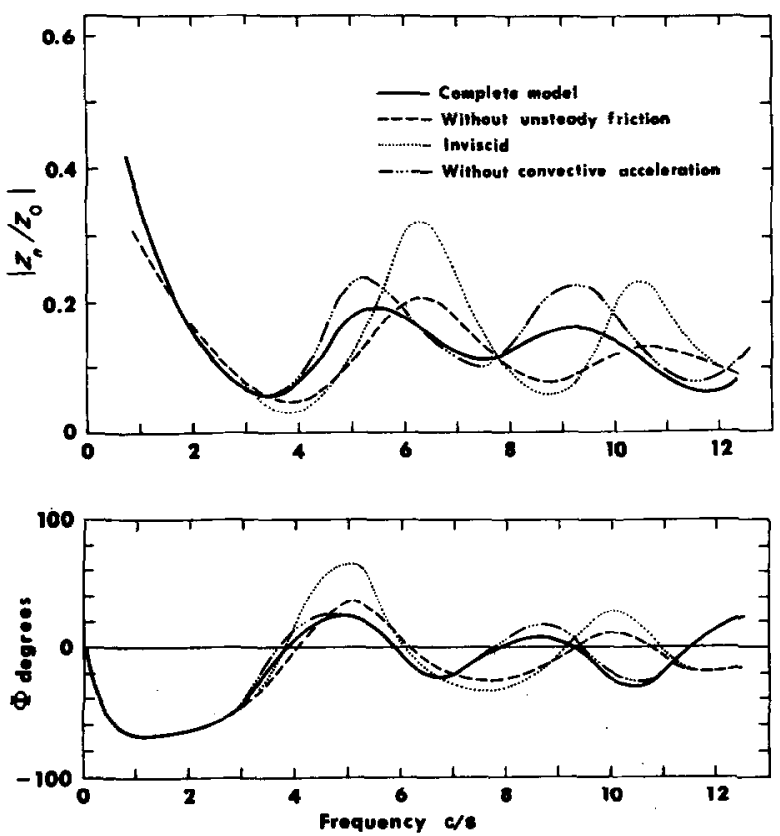

Fig. 16. Normalized input impedance of femoral artery (Site 6), comparing the effects of ignoring unsteady fuid friction, all fluid friction, (inviscid case) and convective acceleration. 


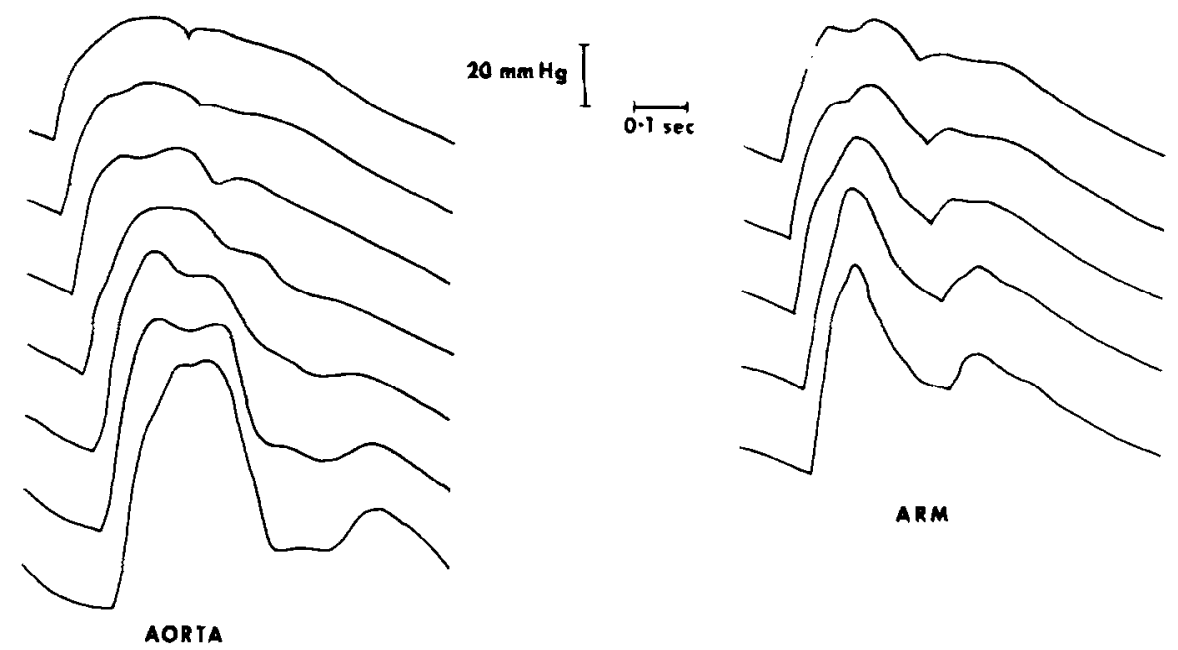

Fig. 17. Pressure pulse mappings in the aorta and the subclavian-radial arteries for the nonlinear model, ignoring unsteady fluid friction.

examine the effects of the frequency dependent friction term used in the nonlinear model.

Setting $\beta$ equal to 1.0 in equation (6) is equivalent to assuming a quasi-steady, laminar, frequency independent fluid resistance. Pulse mappings for the model under this condition are shown in Fig. 17. Clearly, the elimination of frequency dependent friction from the model produces only small differences in the results. Comparison of the input impedance relations shown in Figs. 15 and 16 for the complete model and for the model without the unsteady friction term also confirms the relative insignificance of this factor in determining the overall behavior of the model. In fact, fluid energy dissipation of any kind is apparently of small consequence in the model. By keeping $\beta$ equal to 1.0 and setting the fluid viscosity equal to zero, all fluid energy dissipation effects are removed except at the lumped vessel terminations. In this condition, it is implicitly assumed that the blood is inviscid and that all energy dissipation takes place in the terminal resistance beds. The resulting pulse waveforms for this state are plotted in Fig. 18 and the corresponding impedances derived from the model are shown in Figs. 15 and 16. It can be concluded that since the total absence of local dissipative mechanisms in the model does not change its overall behavior appreciably, these same mechanisms are probably of secondary importance in determining the behavior of the real arterial system.

It has been demonstrated that the distinctive behavior of the model is affected only slightly by vascular energy dissipation and convective acceleration effects. The mid-frequency disparity between the impedance and pulse waveform results from the linear and nonlinear models can then only be attributed to the constraints imposed on the longitudinal impedance in the process of linearization. To arrive at a linear mathematical description of the pressure-flow relationship, boundary conditions at the fluid-vessel interface are linearized by the assumption that the wall displacements are sufficiently small to allow their evaluation at the mean radial position of the wall. Consequently, the longitudinal impedance is rendered independent of vessel distensibility, and, in fact, is the same as that derived for a rigid tube. In contrast, by retaining the vessel cross-sectional area as an independent variable, the longitudinal impedance of the nonlinear model is coupled 

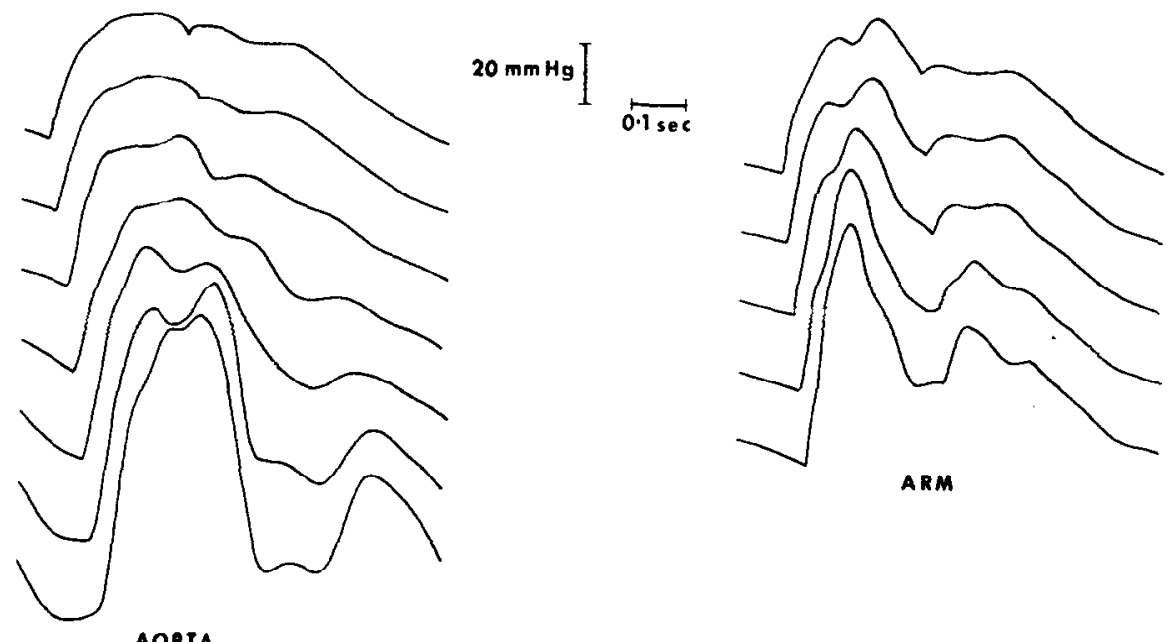

Fig. 18. Pressure pulse mappings in the aorta and the subclavian-radial arteries for the nontinear model, ignoring fluid friction entirely.

with the transverse impedance through the vessel distensibility.

At very low frequencies, fluid inertiance is not a critical factor in the longitudinal impedance, so the linear and nonlinear model impedances correspond. At higher frequencies, fluid inertiance becomes important and this is where the models deviate. Over the frequency range examined in the impedance graphs of Figs. 9 and 10, larger differences between the models are seen in the more distensible aorta than in the femoral artery. Here the larger wall displacements are expected to produce greater fluctuations in longitudinal impedance, which is manifested in the more severe deviation between the models. At the femoral site, the vessel walls are less distensible and the interaction of longitudinal and transverse impedances is expected to be smaller.

The source of the high frequency oscillations initiated by the incisura in the linear model cannot be ascribed to the absence of distensibility effects in the fluid inertiance. A more probable explanation appears to involve the lumped parameter aspects of the linear model. The harmonic frequency associated with the incisura is in the neighbor- hood of $200 \mathrm{c} / \mathrm{s}$, well in excess of the cutoff frequency of the linear model inlet section. The wave length of this disturbance, based on the true phase velocity, is then on the same order of magnitude as the lumped segment length. It is well known that the behavior of lumped electrical low-loss delay lines in the frequency range beyond the cutoff frequency (stop band) is highly reactive, with comparatively small amounts of energy being transmitted. Most of the input energy is then locally oscillatory, and the behavior of the delay line is completely different from that of the continuous line at the same frequencies (Magnusson, 1965). By this analogy, it is likely that the unnatural high frequency oscillations in the linear model are due to these limitations of the lumping procedure.

It would appear, on the basis of the work presented here, that consideration of the nonlinear effects of vessel wall displacements is important in the modeling of pulse wave transmission in the aorta and its immediate branches. In this region, the linear and nonlinear models yield significantly different results. The stiffer peripheral vessels, however, are reasonably represented by linear models. 
For the system as a whole, linear models are capable of realistic prediction of pulse wave transmission, since the characteristic behavior of the propagated waves is exhibited mainly by the lower frequency harmonic components which are affected to a lesser extent by the non-linearities of the system. The nonlinear model does represent a definite improvement over the linear, lumped parameter version. Its behavior is more consistent with physiological measurements over a wider range of frequencies.

Acknowledgements - This work was supported in part by Grant GB 5874 from the National Science Foundation, U.S. Public Health Service Grants AM 10395 and 521 GM 01289, and Research Career Development Award 1 K04 GM 19289 from the National Institute of General Medical Sciences.

\section{REFERENCES}

Abbott, M. B. (1966) Introduction to the Method of Characteristics, American Elsevier, New York.

Attinger, F. M. L. (1968) Two-dimensional in-vitro studies of femoral arterial walls of the dog, Circulation Res. 22, 829-840.

Barnard, A. C. L., Hunt, W. A., Timlake, W. P. and Varley, E. (1966) A theory of fluid flow in compliant tubes, Biophys. J. 6, 717-724.

Gabe, I. T., Karnell, J., Porje, I. G. and Rudewald, B. (1964) The measurement of input impedance and apparent phase velocity in the human aorta, Acta. physiol. scand. 61, 73-84.

Jager, G. N., Westerhof, N. and Noordergraaf, A. (1965) Oscillatory flow impedance in an electrical analog of arterial system, Circulation Res. 16, 121-133.

Kroeker, E. J. and Wood, E. H. (1955) Comparison of simultaneously recorded central and peripheral arterial pressure pulses during rest, exercise and tilted position in man, Circulation Res. 3, 623-632.

Magnusson, B. C. (1965) Transmission Lines and Wave Propagation. Allyn and Bacon, Boston.

Noordergraaf, A. (1963) An analog computer for the human systemic circulatory system, Circulatory Analog Computers (Edited by A. Noordergraaf, G. N. Jager and N. Westerhof). North Holland, Amsterdam.

Noordergraaf, A. (1968) Hemodynamics, in Biological Engineering (Edited by H. P. Schwan), McGraw-Hill, New York.

O'Rourke, M. F., Blazek, J. V., Morreels, C. L., Jr., and Krovet., L. J. (1968) Pressure wave transmission along the human aorta, Circulation Res. 23, 567-579.

Patel, D. J. and Austen, W. G. (1964) Impedance of certain large blood vessels in man, Ann. N.Y. Acad. Sci. 115, 1129-1139.

Patel, D. J., Greenfield, J. C., Jr., Austen, W. G., Morrow, A. G. and Fry, D. L. (1965) Pressure-flow relationships in the ascending aorta and femoral artery of man, J. appl. Physiol. 20, 459-463.
Patel, D. J., Greenfield, J. C., Jr. and Fry, D. L. (1966)

In vivo pressure-length-radius relationship of certain blood vessels in man and dog, in Pulsatile Blood Flow, (Edited by E. O. Attinger), McGraw-Hill, New York.

Pater, L. de, (1966) An electrical analogue of the human circulatory system, Thesis, Univ. Gronigen.

Rockwell, R. L. and Anliker, M. (1969) Shock waves and other nonlinear phenomena of wave propagation in blood vessels, Proc. 8th Intl. Conf. med. biol. Engng., Chicago.

Schaaf, B. W. (1971) Digital computer simulation of human systemic arterial pulse wave transmission: A nonlinear model, $\mathrm{Ph}$. D. Thesis, Univ. of Michigan.

Skalak, R. and Stathis, T. C. (1967) A comparison of linear and nonlinear theories of blood flow, in Proc. $7 \mathrm{th}$ Intl. Conf. Med. Biol. Engng., Stockholm.

Snyder, M. F., Rideout, V. C. and Hillestad, R. J. (1968) Computer modeling of the human systemic arterial tree, J. Biomech. 1, 341-353.

Spencer, M. P. and Denison, A. B. (1959) The squarewave electro-magnetic flowmeter: Theory of operation and design of magnetic probes for clinical and experimental applications, I. R. E. Trans. med. Elert. 6, 220-228

Streeter, V. L., Keitzer, W. F. and Bohr, D. F. (1963) Pulsatile pressure and flow through distensible vessels, Circulation Res. 13, 5-20.

Streeter, V. L., Keitzer, W. F. and Bohr, D. F. (1966) Energy dissipation in pulsatile flow, in Pulsatile Blood Flow, (Edited by E. O. Attinger) McGraw-Hill. New York.

Taylor, M. G. (1965) Wave travel in a nonuniform transmission line in relation to pulses in arteries, Phys. med. Biol. 10, 539-550.

Westerhof, N. (1968) Analog studies of human systemic arterial hemodynamics, Ph. D. Thesis, Univ. of Pennsylvania.

Wiggers, C. J. (1952) Circulatory Dynamics, Grune and Stratton, New York

Womersley, J. R. (1957) An elastic tube thcory of pulse transmission and oscillatory flow in mammalian arteries, W. A. D. C. Rept. No. TR 56-614.

\section{NOMENCLATURE}

$A_{0}$ cross-sectional area of vessel lumen at zero transmural pressure, $\mathrm{cm}^{2}$

$A$ cross-sectional area of vessel lumen, $\mathrm{cm}^{2}$

$\vec{A}$ cross-sectional area of vessel lumen at mean arterial pressure, $\mathrm{cm}^{2}$

$A_{v}$ aortic valve area, $\mathrm{cm}^{2}$

$C$ aortic valve discharge coefficient

$D$ vessel diameter, $\mathrm{cm}$

$E$ modulus of elasticity, dynes $/ \mathrm{cm}^{2}$

$H$ piezometric pressure, $\mathrm{mm} \mathrm{Hg}$

$H_{b}$ terminal bed pressure, $\mathrm{mm} \mathrm{Hg}$

$H_{n}$ modulus of $n^{\text {th }}$ pressure harmonic, $\mathrm{mm} \mathrm{Hg}$

$H_{0}$ mean arterial pressure, $\mathrm{mm} \mathrm{Hg}$

$K$ lateral outflow coefficient, $\mathrm{ml} / \mathrm{mm} \mathrm{Hg} / \mathrm{cm}$

$L$ vessel length, $\mathrm{cm}$

$Q$ volume flow rate, $\mathrm{ml} / \mathrm{sec}$

$Q_{n}$ modulus of $n^{\text {th }}$ flow harmonic, ml

$Q_{0}$ mean arterial flow rate, $\mathrm{ml}$

$R_{r}$ terminal resistance, $\mathrm{mm} \mathrm{Hg} / \mathrm{ml} / \mathrm{sec}$ 
$T$ period associated with heart rate, sec

$V$ instantaneous fluid velocity averaged over vessel cross-section, $\mathrm{cm} / \mathrm{sec}$

$Z_{0}$ vascular resistance, $\mathrm{mm} \mathrm{Hg} / \mathrm{ml} / \mathrm{sec}$

$Z_{n}$ modulus of $n^{\text {th }}$ component of impedance, $\mathrm{mm}$ $\mathrm{Hg} / \mathrm{ml} / \mathrm{sec}$

$\mathscr{C}$ lumped arterial compliance/unit length, $\mathrm{cm}^{2} / \mathrm{mm} \mathbf{~ g g}$

$\mathscr{L}$ lumped fluid inertiance/unit length, $\mathrm{mm} \mathrm{Hg} \cdot \mathrm{sec}^{2} / \mathrm{cm}^{4}$

$\mathscr{W}$ lumped vascular resistance/unit length, $\mathrm{mm} \mathrm{Hg}$. $\mathrm{sec} / \mathrm{cm}^{4}$

$a_{0}$ wave speed, $\mathrm{cm} / \mathrm{sec}$

$f_{n}$ harmonic frequency, cycles/sec

$g$ gravitational constant, $980 \mathrm{~cm} / \mathrm{sec}^{2}$

$h_{0}$ vessel wall thickness at zero transmural pressure, $\mathrm{cm}$

$h$ vessel wall thickness, $c m$

$q$ volume flow rate through vessel wall/unit length, $\mathrm{ml} / \mathrm{cm}$

$r$ radial distance, $\mathrm{cm}$

$t$ time, sec

$v$ total fluid velocity vector, $\mathrm{cm} / \mathrm{sec}$

$v_{z}$ axial component of $v, \mathrm{~cm} / \mathrm{sec}$

$z$ axial coordinate, $\mathrm{cm}$

$\alpha_{n}$ phase of $n^{\text {th }}$ flow harmonic, degrees

$\beta$ momentum flux coefficient

$\beta_{n}$ phase of $n^{\text {th }}$ pressure harmonic, degrees

$\gamma$ ratio of mass density of mercury to the mass density of blood

$\mu$ dynamic viscosity of blood, dyn-sec $/ \mathrm{cm}^{2}$

$\nu$ kinematic viscosity of blood, $\mathrm{cm}^{2} / \mathrm{sec}$

$\rho$ mass density of blood, $\mathrm{g} / \mathrm{cm}^{3}$

$\tau$ dimensionless time, $t / T$

$\tau_{0}$ fluid shear stress at vessel wall, $\mathrm{dyn} / \mathrm{cm}^{2}$

$\phi_{n}$ phase of $n^{\text {th }}$ component of impedance, deg

$\omega$ angular frequency of fundamental harmonic, $\mathrm{rad} / \mathrm{sec}$ 